den Gasgesetzen im Endresultat sich gerade herausheben müssen.

Man kann diesen Gedanken weiter verfolgen: Man gelangt ${ }^{1}$ ) dann sehr leicht $z$ a allerhand Beziehungen, wie $z$. B. der folgenden:

$\frac{L_{\mathrm{H}_{g} \mathrm{Cl}}: L_{T l C l}: L_{A g C l}: L_{C u} \mathrm{Cl} \ldots}{L_{H_{\mathrm{B} B r}}: L_{T l B r}: L_{A g B r}: L_{C u B r}}=$ konst., d. h. unabhängig vom Lösungsmittel. Mit $L$ ist das sogen. "Löslichkeitsprodukt" bezeichnet. Man kann den Sinn dieser Gleichung am besten erkennen, wenn man fingierte Zahlen für die verschiedenen $L$ 's einsetzt, doch will ich darauf nicht näher eingehen, sondern nur erwähnen, dass man noch weiter gehen kann: Durch eine, meines Erachtens berechtigte Extrapolation von theoretisch abgeleiteten und experimentell bestätigten Gleichungen ${ }^{2}$ ), kann man

r) Nach Nernst lässt sich die E. K. des Elementes durch die Formel

$$
R T \ln \frac{[T l] H g}{T l \cdot[H g]}
$$

ausdrücken, wo $T l, H g^{*}$ die Konzentrationen des Thallo-, resp. Mercuroions sind, $[\mathrm{Tl}]$ und $[\mathrm{Hg}]$ die "Lösungstensionen" der Metalle. Ferner ist $\mathrm{Tl} \cdot \mathrm{Cl}$, $=\mathrm{LTlCl}_{\mathrm{H}} \mathrm{Hg} \cdot \mathrm{Cl}^{\prime}=\mathrm{LHg}_{\mathrm{g}} \mathrm{Cl}$, wo mit $L$ die ,Löslichkeitsprodukte" bezeichnet sind. Folglich ist

$$
\pi=R T \ln \frac{[T l]}{L T l C l}-\frac{\mathrm{LHgCl}}{[\mathrm{Hg}]}
$$

und dieses unabhängig vom Lösungsmittel. Bildet man eine ähnliche Gleichung für Bromüre und subtrahiert, so fallen $[\mathrm{Tl}]$ und $[\mathrm{Hg}]$ heraus, und man erhält

$$
\frac{L_{H g} C l}{L H_{g B r} B}: \frac{L T l C l}{L T l B r}=\text { konst., }
$$

welche Beziehung sich ohne weiteres verallgemeinern lässt.

2) In einer früheren Arbeit leitete ich die Beziehung $a b$, dass die E. K. an der Berührungsstelle mit einiger Wahrscheinlichkeit schliessen, dass sowohl der Nenner, wie der Zähler der linken Seite der obigen Gleichung jeder für sich unabhängig von dem Lösungsmittel ist, $d$. h. dass das Verhältnis der Lösungsprodukte verschiedener schwerlöslicher Salze unabhängig vom Lösungsmittel oder in allen Lösungsmitteln dasselbe ist.

$z$ weier im Verteilungs-Gleichgewicht stehender Lösungen desselben Flektrolyten in zwei verschiedenea Lösungsmitteln unabhängig von der Konzentration sein müsse. Diese Folgerung wurde durch den Versuch bestätigt. Wenn nun diese Unabhängigkeit von Konzentration auch für unendlich verdünnte Lösungen, $d . h$. die reinen Lösungsmittel gilt, so muss, wie ich jetzt glaube, die E. K. nur von der Natur der Lösungsmittel abhängen und unabhängig von der des sich verteilendesn Elektrolyten sein. Auch dieser Schluss wurde rein experimentell in der obigen Arbeit gefunden. Führt man nun diese Beziehung in die dort entwickelten Gleichungen ein, so ergeben sich u. a. folgende Resultate: Das Verhältnis der Lösungstensionen verschiedener Metalle ist unabhängig vom Lösungsmittel, oder das Teilungsverhältnis sämtlicher, z. B. einwertiger Kationen zwischen zwei Lösungsmitteln ist gleich (aber im allgemeinen verschieden von dem konstanten Teilungsverhältnis sämtlicher einwertiger Anionen). Die verschiedenen Lösungsmittel haben also ein verschiedenes „Lösevermögen" für positiv, resp. negativ geladene Stoffe.

Setzt man die eben entwickelte Beziehung $\frac{[T l]}{[H g]}$ - konst. in die in Note I gegebene Gleichung

$$
\frac{[T l]}{L T / C l} \cdot \frac{L_{H g C l}}{[H g]}=\text { konst. }
$$

ein, so erhält man die im Text gegebene Beziehung $\mathrm{LHgCl}_{\mathrm{H}} \mathrm{LTlCl} \ldots=$ konst. (unabhängig vom Lösungsmittel).

\title{
Diskussion.
}

Auf Vorschlag des Vorsitzenden wird die Diskussion über Normalelemente auf den folgenden Tag verschoben, da noch ein Vortrag von
Herrn Cohen-Amsterdam für dieses Thema vorgesehen ist.

Vorsitzender: Ich bitte Herrn Professor Küster, das Wort zu ergreifen.

Herr Prof. Dr. F. W. Küster-Clausthal:

\section{ÜBER DAS ELEKTROCHEMISCHE VERHALTEN DES SCHWEFELS.}

Nach Versuchen des Herrn Dr. Woldemar Hommel ${ }^{\mathbf{1}}$.

Vor einigen Jahren hatte ich bei der Elektrolyse von Polysulfidlösungen beobachtet, dass die mit der Zersetzungszelle in üblicher Weise ver-

1) Die Arbeit wird ausführlich in der ,Zeitschrift für anorganische Chemie" erscheinen. bundenen Volt - und Ampèremeter merkwürdige, periodisch regelmässig wiederkehrende Schwankungen der Stromstärke und der Klemmspannung anzeigten. Der Verlauf dieser Schwankungen ergab nun eine überraschende Aehnlichkeit mit den Schwankungen der Lösungsgeschwindigkeit 
des "schwingenden" Chroms in Säuren, uber welche W. Ostwald auf der Münchener Naturforscher-Versammlung so interessante Mitteilungen machte. Trotz eifrigster und scharfsinnigster Bearbeitung hat das "schwingende" Chrom sich sein Geheimnis noch nicht entreissen lassen, es schien deshalb von doppeltem Interesse, der schwingenden Sulfidlösung etwas mehr Aufmerksamkeit zu schenken, zumal bei letzterer der Grund der Stromschwächungen klar zu Tage lag: ein periodisch auftretender und wieder verschwindender Ueberzug von Schwefel auf der Anode. Ganz analog konnte das sich unter Wasserstoffentwicklung in Säuren lösende Chrom periodisch von Wasserstoffschichten eingeschlossen werden, die den Zutritt der Säure erschweren, von Zeit zu Zeit aber durch Zerreissen zu getrennten, sich rasch ablösenden Blasen der Säure wieder ausgedehnteren Zutritt gestatten. Wenn die Gründe für das Schwingen des Chroms und der Sulfidlösung wirklich so ganz analoge waren, so musste auch der Verlauf der Perioden selbst grosse Aehnlichkeit aufweisen. Ich habe deshalb durch K. Koelichen die Stromstärken bei der Sulfidelektrolyse mit Hilfe eines zweckmässig abgeänderten Ostwaldschen selbstthätigen Registrierapparates graphisch darstellen lassen, und es haben sich hierbei Kurven ergeben ${ }^{1}$ ), die in der That den Chromlösungskurven zum Verwechseln ähnlich sehen ${ }^{2}$ ). Es liess sich nun zeigen, dass der Grund des Phänomens bei der Elektrolyse der Sulfidlösung in der That einzig und allein der veränderte Widerstand an der Anode war, indem der Schwefelüberzug isolierend wirkte. Wenn sich nun auch dieser ursächliche $\mathrm{Zu}$ sammenhang zwischen Stromschwächung und Schwefelabscheidung mit Sicherheit nachweisen liess, so war und blieb doch die periodische Abscheidung des Schwefels mit abwechselnder Wiederauflösung etwas sehr Rätselhaftes. Denn es lässt sich zunächst gar nicht einsehen, warum der Vorgang periodisch erfolgt, man müsste vielmehr annehmen, dass sich ein stationärer $\mathrm{Zu}$ stand ausbildet, indem sich zwischen Abscheidungsund Auflösungsgeschwindigkeit ein Gleichgewicht

г) Zeitschr. f. Elektrochemie 7, $63^{\text {r. }}$

2) Zeitschr. f. physik. Chemie 35, $33-76$ und 204-256; vergl. besonders Fig. 9 auf Seite 235 . herstellt Die Periodizität des Vorganges hat deshalb in hohem Grade das Interesse der Fachgenossen wachgerufen ${ }^{1}$ ), so dass es lohnend erscheint, ihrem Grunde sorgfältig nachzuspüren. Es ist deshalb die Auflösung von Schwefel in Sulfiden ebenso eingehend und sorsfältig von den verschiedensten Gesichtspunkten aus zu studieren, wie die Abscheidung auf elektrolytischem Wege. Was man bisher über diese Vorgänge weiss, ist äusserst wenig, es beschränkt sich fast nur auf die Angaben, wieviel Schwefel sich überhaupt in Sulfidlösungen noch weiter zu lösen vermag, und selbst diese Angaben erscheinen nicht alle so ganz zuverlässig. Deshalb wurden in den letzten Semestern im chemischen Institut der Bergakademie zu Clausthal Untersuchungen über die Auflösung von Schwefel in Sulfiden und Polysulfiden von verschiedenen Gesichtspunkten aus in Angriff genommen, und zwar untersuchte Herr Dr. Carol Koelichen die Geschwindigkeit der Auflösung, Herr Eduard Heberlein die Menge des gelösten Schwefels, die Hydrolyse der verschiedensten Lösungen und anderes, während Herrn Leslie Jonas und Herrn Dr. W. Hommel die Bearbeitung der Sulfid - und Polysulfidlösungen von elektrochemischem Standpunkte aus übertragen wurde. In folgendem will ich nun ganz kurz über die von Herrn Dr. Hom mel erhaltenen Resultate berichten.

Für die Geschwindigkeit, mit welcher sich Schwefel in Schwefelnatrium-Lösung auflöst, wird ausser anderen Faktoren auch die Menge des Schwefels bestimmend sein, welcher in der Lösung schon vorhanden ist; denn die Geschwindigkeit eines jeden Vorganges ist im allgemeinen proportional seinem Abstande von der Gleichgewichtslage 2). Nun ist aber der vom Schwefelnatrium aufgenommene Schwefel in der Lösung in sehr verschiedenen Zuständen vorhanden, zum weitaus überwiegenden Anteile zweifelsohne an die Schwefelionen addiert als Bestandteil der komplexen Anionen $S_{2}{ }^{\prime \prime}, S_{3}{ }^{\prime \prime}$, $S_{4}{ }^{\prime \prime}$ und $S_{5}{ }^{\prime \prime}$; in kleiner Menge sicher auch als "freier" Schwefel $S_{x}$, worin das $x$ gleich 2 zu setzen ist, da der "freie" Schwefel infolge der ausserordentlich grossen Verdünnung prak-

1) Verg1. Zeitschr. f. Elektrochemie 7, 633-635.

2) Vergl. W. Ostwald: "Grundlinien ", S. I30. 
tisch vollständig in die kleinsten Molekeln zerfallen ist. Direkt ausschlaggebend für die Geschwindigkeit, mit welcher der Schwefel von der Schwefelnatrium-Lösung aufgenommen wird, kann nur der gelöste, noch nicht durch weitere Umsetzung (Polysulfidbildung) veränderte Schwefel, der "freie" Schwefel sein, über dessen Konzentration sich aber zunächst nichts aussagen lässt. Man wird aber wenigstens über das Verhältnis dieser Konzentrationen in den verschiedenen Lösungen etwas erfahren aus dem Potential, das eine Platinelektrode in den fraglichen Lösungen annimmt, denn es ist schon mehrfach nachgewiesen worden ${ }^{1}$ ), dass auch auf Metalloïde die Nernstsche Gleichung:

$$
\pi=\frac{R T}{n} \ln \frac{p}{p}
$$

anwendbar ist.

In dieser Gleichung bedeutet, wie üblich, $\pi$ das Potential, $R$ die Gaskonstante, $T$ die Temperatur in absoluter Zählung, $n$ die Wertigkeit des Ions, hier beim Schwefel gleich zwei, $P$ den Losungsdruck des Schwefels und $p$ den osmotischen Druck der Schwefelionen. $P$ und $p$ sind unbekannt; denn $P$ ist zwar der Konzentration des freien Schwefels $S_{2}$ proportional, diese kennen wir aber nicht, und auch $p$ ist nicht genügend sicher bekannt, da infolge sehr weit gehender Hydrolyse relativ grosse Unsicherheit der Kenntnis des unveränderten und normalionisierten Schwefelnatriums in der Lösung besteht. Nichtsdestoweniger können wir uns Gewissheit über die relativen Grössen von $P$ in den verschiedensten Lösungen verschaffen, und nur auf relative Grössen kommt es uns an, da ja, wie schon oben hervorgehoben, nur der relative Abstand der $P$-Werte in den verschiedenen Lösungen vom Maximal-P.Werte für die Geschwindigkeit des Lősungsvorganges ausschlaggebend ist. Die Grundlage für die Herleitungen sollen uns die Potentiale liefern, die Platin- und Silberelektroden in verschieden konzentrierten, mit verschiedenen Mengen Schwefel versetzten und auch sonst variierten Lösungen von Schwefelnatrium annehmen. Ueber diese Potentiale soll deshalb zunächst Mitteilung gemacht werden.

I) Siehe z. B. F. Crotogino: „Studien über Oxydationspotentiale", Dissertation, Giessen rgoa.
Am einfachsten liegen die Verhältnisse bei den Lösungen, welche für Schwefel gesättigt sind, denn sie sind ja in Bezug auf den "freien" Schwefel alle gleich konzentriert, mit Ausnahme vielleicht der konzentriertesten, welche für „freien" Schwefel ein etwas anderes Lősungsverhältnis haben könnten, indem ein so grosser Gehalt an Natriumpolysulfid schon eine nicht zu vernachlässigende Aenderung des Mediums bedeutet. Da die Lösungen für "freien" Schwefel gesättigt sind, so ist in ihnen allen der Ionisationsdruck des Schwefels gleich, das $P$ in der Nernstschen Formel konstant. Die Potentiale geben demnach Aufschluss über die relativen Grössen von $p$.

Platinelektroden in mit Schwefel gesättigten Lösungen von Schwefelnatrium verschiedener Konzentration zeigten gegen die Normalelektrode folgende Spannungen:

\begin{tabular}{|c|c|c|c|c|c|}
\hline $\mathrm{Nr}$. & $\begin{array}{l}\text { Normalităt } \\
\text { des } \mathrm{Na}_{2} S_{x}\end{array}$ & $\begin{array}{l}\text { Wert des } x \\
\text { in } N a_{2} S_{x}\end{array}$ & $\begin{array}{c}\pi \\
\mathrm{m} V\end{array}$ & $\begin{array}{c}\text { Differenz } \\
\mathrm{m} \mathrm{V}\end{array}$ & $\begin{array}{l}\text { Differenz } \\
\text { berechnet } \\
\text { m V }\end{array}$ \\
\hline $\begin{array}{r}1 \\
2 \\
3 \\
4 \\
5 \\
6 \\
7 \\
8 \\
9 \\
10\end{array}$ & $\begin{array}{l}4 \\
2 \\
1 \\
1 / 2 \\
1 / 4 \\
1 / 8 \\
1 / 18 \\
1 / 32 \\
1 / 64 \\
1 / 128\end{array}$ & $\begin{array}{l}4,47 \\
4,67 \\
4,84 \\
4,98 \\
5,12 \\
5,22 \\
5,24 \\
5,20 \\
5,04 \\
4,45\end{array}$ & $\begin{array}{l}621,1 \\
608,7 \\
600,0 \\
591,6 \\
583,6 \\
575,8 \\
568,3 \\
560,3 \\
55^{2,3} \\
54^{x, 1}\end{array}$ & $\begin{array}{r}\mathrm{I} 2,4 \\
8,7 \\
8,4 \\
8,0 \\
7,8 \\
7,5 \\
8,0 \\
8,0 \\
\mathrm{II}, 2\end{array}$ & $\begin{array}{l}7, I \\
7,7 \\
8,0 \\
8,2 \\
8,5 \\
8,5 \\
8,5\end{array}$ \\
\hline
\end{tabular}

Aus der weitgehenden Uebereinstimmung zwischen den in bekannter Weise berechneten und gefundenen Zahlen folgt, dass auch bei der Schwefelelektrode die herrschenden Verhältnisse durch die Nernstsche Formel ihren richtigen Ausdruck finden.

Die folgende Tabelle giebt die Potentiale, die eine Silberelektrode in mit Schwefel gesättigten Schwefelnatrium-Lösungen verschicdener Konzentration annimmt. Die Potentiale der Platinelektrode sind zum Vergleich danebengestellt.

Bei der Messung der Werte für die Silberelektroden, die zum Teil Herr Leslie Jonas ebenfalls im Clausthaler Laboratorium ausgeführt hat, konnte nicht dieselbe zeitraubende Sorgfalt und häufige Wiederholung der Einzelversuche durchgeführt werden, wie bei der Ermittlung der Werte für die Platinelektroden. Die Zahlen sind deshalb hier weniger zuverlässig, namentlich die der verdünnten Loosungen, die gegen 


\begin{tabular}{|c|c|c|c|c|c|c|c|}
\hline $\mathbf{I}$ & 2 & 3 & 4 & 5 & 6 & 7 & 8 \\
\hline Nr. & $\begin{array}{l}\text { Norma- } \\
\text { lität des } \\
N a_{2} S_{x}\end{array}$ & $\begin{array}{c}\text { Wert } \\
\text { des } x \\
\text { in } \\
N a_{2} S_{x} \\
\end{array}$ & $\begin{array}{c}\tau \text { der } \\
\text { Silber- } \\
\text { elektrode } \\
\mathrm{mV}\end{array}$ & $\begin{array}{l}\text { Diffe- } \\
\text { renzen }\end{array}$ & $\begin{array}{c}\pi \text { der } \\
\text { Platin. } \\
\text { elektrode } \\
\mathrm{m} \mathrm{V}\end{array}$ & 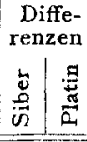 & $\begin{array}{c}\pi \text { für } \\
\text { Silber } \\
\text { berechn } \\
\mathrm{m} \mathrm{V}\end{array}$ \\
\hline I & & & & & 62 & & \\
\hline 2 & 2 & & & 6,5 & $6 c$ & 167,2 & 777. \\
\hline 3 & I & 4,8 & & 5 & 60 & 170,0 & 769 \\
\hline & $1 / 2$ & 4, & 76 & 9 & & 169,3 & 760 \\
\hline & $1 / 4$ & $5, \mathrm{I}$ & 75 & IC & $5^{8}$ & 167 & 742 \\
\hline & 1 & 5,22 & ca. 73 & & $5 ?$ & 163 & 74 \\
\hline & $1 / 10$ & 5,2 & 7 & 8 & & 172 & 73 \\
\hline & & 5,2 & 7 & 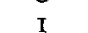 & & 172 & 72 \\
\hline$x$ & 104 & 5,04 & $=73^{1}$ & 30 & 55 & 179 & 721 \\
\hline IC & 128 & 4,45 & 70 & & 541 & 160 & 710, \\
\hline
\end{tabular}

allerhand Einflüsse viel empfindlicher sind, als die konzentrierten. Die geringere Zuverlässigkeit der Zahlen, namentlich bei den verdünnten Lösungen, zeigt sich denn auch sehr deutlich in der Unregelmässigkeit der Differenzen. Dass aber das Potential der Silberelektrode in der That dem der Platinelektrode parallel läuft, zeigt die 7. Spalte, in welcher das Potential SilberPlatin eingetragen ist. Die Werte schwanken mit verhältnismässig nicht grossen Abweichungen um den Mittelwert I69,I. In der 8. Spalte sind die Werte eingetragen, welche sich für die Silberelektrode berechnen, wenn man zum Werte für die Platinelektrode die Durchschnittsdifferenz r69, I addiert. Mit Ausnahme der beiden letzten, ganz unsicheren Werte, weichen die für Silber gefundenen von den berechneten nicht stark und unregelmässig $a b$, so dass der Parallelismus der Silber- und der Platinwerte in der That nachgewiesen ist.

Weiterhin wurden nun auch die Potentiale von Platin- und Silberelektroden in Sulfid- lösungen gemessen, die für Schwefel nicht gesättigt waren. Die Potentiale in diesen Lösungen zeigten z. B. grosse Veränderlichkeit, und zwar um so mehr, je weniger über die Zusammensetzung $\mathrm{Na}_{2} \mathrm{~S}$ überschiessender Schwefel vorhanden war. Die grösste Veränderlichkeit zeigt demnach die reine Monosulfidlösung $\mathrm{Na}_{2} S$ selbst. Es ist dies zu vergleichen mit dem Verhalten der Wasserstoff - und Sauerstoffelektrode in den ,"neutralen “ Lösungen. Diese sind eben gerade so wenig für Wasserstoff- und Hydroxylionen definiert, wie die „reine" Monosulfidlösung für freien Schwefel. In folgender Uebersicht sind die Potentiale der Platin- und Silberelektrode in normalen $N a_{2} S_{x}$-Lösungen zusammengestellt.

\begin{tabular}{|c|c|c|c|c|c|c|}
\hline I & 2 & 3 & 4 & 5 & 6 & 7 \\
\hline Nr. & $\begin{array}{l}\text { Wert } \\
\text { des } x \text { in } \\
N a_{2} S_{x}\end{array}$ & $\begin{array}{c}\pi \text { der } \\
\text { Silber } \\
\text { elektrode }\end{array}$ & $\begin{array}{l}\text { Diffe- } \\
\text { renz }\end{array}$ & $\begin{array}{c}\pi \text { der } \\
\text { Platin- } \\
\text { elektrode }\end{array}$ & $\begin{array}{l}\text { Diffe- } \\
\text { renz }\end{array}$ & $\begin{array}{c}\pi \text { Silber } \\
\text { minus } \\
\pi \text { Platio }\end{array}$ \\
\hline I & I & 920 & & 799 & & I2I \\
\hline 2 & 1,25 & 909 & & 787 & 12 & 122 \\
\hline 3 & $I, 5$ & 895 & 4 & 766 & 21 & 129 \\
\hline 4 & 2 & 893 & 20 & $75^{\circ}$ & TO 5 & 143 \\
\hline 5 & 3 & 873 & 50 & 730,5 & $\begin{array}{l}19.5 \\
532\end{array}$ & $I_{42,5}$ \\
\hline 6 & & 823 & $\begin{array}{l}50 \\
53\end{array}$ & 677,3 & $\begin{array}{l}3,3 \\
77,3\end{array}$ & 145,7 \\
\hline 7 & 4,84 & $77^{\circ}$ & & 600,0 & & 170,0 \\
\hline
\end{tabular}

Die Zahlen sollen nur als vorläufige gelten, sie genügen jedenfalls für eine erste Orientierung, zumal für das Verhältnis der Platin- zur Silberelektrode. Da beide Elektroden immer zugleich in denselben Lösungen stehend gemessen wurden, so dürften die Differenzen $\pi$ Silber minus $\pi$ Platin als ziemlich zuverlässig gelten, zumal fast immer die oft beobachteten Schwankungen an beiden Elektroden zugleich und parallel verlaufend auftraten.

\section{Diskussion.}

Herr Cohen-Amsterdam: Ich erlaube mir, zu dem Vortrage des Herrn Vorredners zu bemerken, dass ich vor einiger Zeit mit Herrn Dr. Ringer eine Beobachtung gemacht habe, die Sie vielleicht interessieren wird. Vielleicht hat er auch Gelegenheit, derartige Untersuchungen fortzusetzen; vielleicht ist die Sache auch bekannt. Wenn man Wismut in eine salpetersaure Lösung bringt und elektrolysiert diese Lösung, so sieht man sehr starke Schwankungen auftreten, sowohl am Voltmeter, wie am Ampèremeter. Ist Ihnen das vielleicht bekannt?

Herr Küster-Clausthal: Ja, in meinem Laboratorium sind noch von Herrn Dr. von Stein- wehr und anderen eine grosse Anzahl von solchen Beobachtungen gemacht worden, und da haben wir nachher in der Litteratur gefunden, dass das meiste von dem, was wir da gefunden haben, schon längst bekannt ist, dass zum Teil schon sehr alte, derartige Beobachtungen vorliegen von vor 50 Jahren. Es ist schon sehr viel darüber bekannt, aber es ist in der alten Litteratur verstreut, und uns war das unbekannt; namentlich hat Schoenbein schon viele solche Beobachtungen gemacht. Unter gewissen Umständen sieht man an Quecksilberelektroden wunderschön, wie die blanke Oberfläche allmählich verschwindet. Dann huscht plötzlich 
ein Lichtschein darüber, und es giebt gleichzeitig an Strom- und Spannungsmesser eine Schwankung, genau so, wie bei der Elektrolyse der Schwefelverbindungen.

Herr Foerster-Dresden: Die Beobachtung, welche eben Herr Kollege Cohen erwähnte, sind bezüglich der analytischen Bestimmung des Kupfers mir auch wohl bekannt in stark salpetersaurer Lösung. Ich habe mir die Vorstellung gebildet, dass es sich dabei um Reduktion der Salpetersäure zum Ammoniak handelt. Die Erscheinungen treten besonders auf bei hohen Stromdichten; es bildet sich dann eine konzentrierte Lösung von Ammoniak um die Kathode, welche plötzlich, indem sich Wasserstoff entwickelt, durchbrochen wird, während die Stromstärke steigt und die Spannung sinkt. Dann beginnt der Stromrückgang und Spannungs- anstieg von neuem, und man kann die Schwankungen, so oft man will, sich wiederholen sehen.

Herr Küster-Clausthal: Ich möchte hinzufügen, dass man das auch unter Umständen hören kann, gar nicht zu sehen braucht. Wenn man eine mit Chlorsilber formierte Silberelektrode reduziert, dann beobachtet man anfangs keine Wasserstoff-Entwicklung, nachdem das Chlorsilber vollkommen reduziert ist, und dann auf einmal tritt Wasserstoff auf mit solcher Plötzlichkeit, dass es förmliche kleine Explosionen sind, man hört es direkt knistern, dann giebt es am Ampèremeter und Voltmeter einen Ausschlag. Also das sind Uebersättigungserscheinungen. Ich glaube, das ist alles auf dieselbe Ursache zurückzuführen.

Herr K. Kellner-Hallein:

\section{ÜBER DAS VERHALTEN VON BROM GEGEN ENTLADUNGEN HOCHGESPANNTER ELEKTRISCHER STRÖME.}

Ich will hier in Kürze über das Verhalten von Brom gegen Entladungen hochgespannter elektrischer Ströme berichten.

Die theoretischen Erwägungen, die mich veranlasst haben, solche Versuche anzustellen, werde ich gelegentlich in unserer Zeitschrift für Elektrochemie veröffentlichen und mich heute nur darauf beschränken, Ihnen die praktischen Resultate dieser Versuche vorzulegen.

Wie Ihnen bekannt sein dürfte, habe ich vor beiläufig 8 Jahren gefunden, dass reines, trockenes Chlorgas beim Durchleiten hochgespannter elektrischer Ströme Eigenschaften annimmt, welche dem gewöhnlichen Chlorgas nicht zukommen. Es bildet z. B. auch im Dunkeln die Chloressigsäuren, welche gewöhnliches Chlorgas nur unter der Einwirkung des direkten Sonnenlichtes liefert, u. s. w.

Meine angestrengten Berufsarbeiten und die damit verbundenen zahlreichen und weiten Reisen haben es mir bis heute unmöglich gemacht, die Resultate meiner diesbezüglichen Versuche mit Chlor so ausführlich zu veröffentlichen, als ich dies gewünscht hătte.

Der Gegenstand hat mich aber derart interessiert, dass ich die wenigen freien Minuten, die mir beschieden sind, zu Hilfe nahm, um ähnliche Versuche auch mit anderen Körpern durchzuführen.

Bis jetzt habe ich Brom, Jod und Schwefel in Arbeit genommen und freue mich nun, Ihnen über das Brom zu referieren.

Das zu den Versuchen verwendete Brom wurde als "chemisch rein" von Merck bezogen, nach Pierre weiter gereinigt, hierauf einer fraktionierten Destillation unterworfen und nur das bei der mittleren Fraktion übergegangene Destillat zu den Versuchen verwendet.

Die getrockneten Dämpfe dieses Körpers wurden in Glasgefäsen kondensiert, welche nach Art der Siemensschen Ozonrohre doppelwandig und aus bleifreiem Glas angefertigt worden sind. Dieselben hatten entweder die Form I oder II (Fig. I66).

Die seitlichen Röhrchen $a$, bezw. $a^{\star}$ wurden mit den Röhrchen des den getrockneten Bromdampf liefernden Apparates verschmolzen, während $b$, bezw. $b^{\prime}$ mit einer Luftpumpe in Verbindung standen.

Beide Leitungen konnten durch Glashähne, welche sowohl am Destillierkolben, als auch an 